\title{
Distribution of bound and free water in anatomical fractions of pine residues and corn stover as a function of biological degradation
}

Ling Ding ${ }^{\mathrm{a}}$, Josephine Gruber ${ }^{\mathrm{b}}$, Allison E. Ray ${ }^{\mathrm{c} *}$, Bryon S. Donohoe ${ }^{\mathrm{b} *}$, and Chenlin $\mathrm{Li}^{\mathrm{a} \ddagger}$

[a] Energy and Environmental Science and Technology, Idaho National Laboratory, Idaho Falls, ID, 83415, United States

[b] Biosciences Center, National Renewable Energy Laboratory, Golden, CO, 80401, United States

[c] Science and Technology, Idaho National Laboratory, Idaho Falls, ID 83415, United States

[†] current address: Bioenergy Technologies Office, Office of Energy Efficiency and Renewable Energy, U.S. Department of Energy, Washington, D.C. 20585

*Corresponding Authors:

Email: allison.ray@inl.gov; Tel: +208526 4549

Email: byron.donohoe@,nrel.gov; Tel: +303 3847773

Page: 10

Figure: 2

Tables: 7 
Table S1. Transverse relaxation time $\left(\mathrm{T}_{2}\right)$ and peak amplitude $(\mathrm{A})$ of the pine fraction samples.

\begin{tabular}{ccccccc}
\hline & $\begin{array}{c}\text { Anatomical } \\
\text { fractions }\end{array}$ & $\begin{array}{c}\text { Moisture } \\
\text { content } \\
(\%)\end{array}$ & Pool $_{\mathrm{b}}{ }^{*}(\mathrm{~ms})$ & $\mathrm{A}_{\mathrm{b}}{ }^{*}(\%)$ & Pool $_{\mathrm{f}}{ }^{*}(\mathrm{~ms})$ & $\mathrm{A}_{\mathrm{f}}{ }^{*}(\%)$ \\
23-year-old & Bark & 8.25 & $1.33 \pm 0.007$ & $9.30 \pm 0.3$ & $87 \pm 10$ & $21.8 \pm 0.2$ \\
& Branch & 7.53 & $1.05 \pm 0.02$ & $41 \pm 1$ & $64 \pm 9$ & $18.6 \pm 0.2$ \\
& Needle & 7.51 & $0.89 \pm 0.004$ & $6.40 \pm 0.1$ & $48 \pm 4$ & $70.8 \pm 0.4$ \\
13-year-old & Bark & 7.44 & $1.14 \pm 0.01$ & $7.80 \pm 0.3$ & $140 \pm 10$ & $42 \pm 0.6$ \\
& Branch & 8.06 & $1.07 \pm 0.01$ & $12.60 \pm 0.4$ & $90 \pm 10$ & $21.4 \pm 0.3$ \\
& Needle & 7.95 & $0.26 \pm 0.001$ & $3.41 \pm 0.08$ & $53 \pm 5$ & $66.5 \pm 0.4$ \\
\hline
\end{tabular}

*b: bound water; f: free water 
Table S2. Chemical composition (weight percentages) measured for 23 and 13-year-old and degraded anatomical fractions.

\begin{tabular}{llllllllll}
\hline Component (wt \%) & \multicolumn{3}{c}{ 23-year-od } & \multicolumn{3}{c}{ 13-year-old } & \multicolumn{3}{c}{ Degraded } \\
& Bark & Branch & Needle & Bark & Branch & Needle & Bark & Branch & Needle \\
& & & & & & & & \\
water extractives & 6.29 & 3.15 & 22.11 & 11.35 & 2.86 & 23.11 & 2.94 & 3.19 & 2.67 \\
lignin & 50.23 & 35.76 & 25.82 & 47.10 & 35.51 & 27.12 & 51.98 & 42.87 & 47.88 \\
glucan & 18.65 & 32.62 & 18.30 & 17.11 & 33.10 & 19.69 & 17.94 & 25.93 & 19.39 \\
xylan & 3.76 & 8.35 & 2.96 & 3.09 & 8.49 & 3.30 & 3.08 & 7.00 & 3.88 \\
\hline
\end{tabular}


Table S3. Transverse relaxation time $\left(\mathrm{T}_{2}\right)$ and peak amplitude of pine samples with different particle sizes and ages.

\begin{tabular}{lcccc}
\hline & Pool $_{\mathrm{b}}(\mathrm{ms})$ & $\mathrm{A}_{\mathrm{b}}(\%)$ & Pool $_{\mathrm{f}}(\mathrm{ms})$ & $\mathrm{A}_{\mathrm{f}}(\%)$ \\
23-year-old $0.2 \mathrm{~mm}$ & $0.89 \pm 0.004$ & $6.40 \pm 0.1$ & $48 \pm 4$ & $70.8 \pm 0.4$ \\
13-year-old 0.2mm & $0.26 \pm 0.001$ & $3.41 \pm 0.08$ & $53 \pm 5$ & $66.5 \pm 0.4$ \\
23-year-old 0.5mm & $0.62 \pm 0.004$ & $22.80 \pm 0.6$ & $44 \pm 4$ & $101 \pm 0.5$ \\
13-year-old 0.5mm & $0.52 \pm 0.004$ & $6.30 \pm 0.2$ & $53 \pm 5$ & $62.4 \pm 0.4$ \\
\hline
\end{tabular}


Table S4. Transverse relaxation time $\left(\mathrm{T}_{2}\right)$ and peak amplitude of degraded pine residues.

\begin{tabular}{cccccc}
\hline & $\begin{array}{c}\text { Anatomical } \\
\text { fractions }\end{array}$ & Pool $_{\mathrm{b}}(\mathrm{ms})$ & $\mathrm{A}_{\mathrm{b}}(\%)$ & Pool $_{\mathrm{f}}(\mathrm{ms})$ & $\mathrm{A}_{\mathrm{f}}(\%)$ \\
non-degraded & Bark & $1.33 \pm 0.007$ & $9.30 \pm 0.3$ & $87 \pm 10$ & $21.8 \pm 0.2$ \\
& Branch & $1.05 \pm 0.02$ & $41 \pm 1$ & $64 \pm 9$ & $18.6 \pm 0.2$ \\
& Needle & $0.89 \pm 0.004$ & $6.40 \pm 0.1$ & $48 \pm 4$ & $70.8 \pm 0.4$ \\
degraded & Bark & $0.59 \pm 0.008$ & $38 \pm 1$ & $41 \pm 6$ & $14.9 \pm 0.08$ \\
& Branch & $1.01 \pm 0.02$ & $44 \pm 1$ & $60 \pm 10$ & $19.4 \pm 0.2$ \\
& Needle & $0.82 \pm 0.01$ & $47 \pm 2$ & $37 \pm 8$ & $20.2 \pm 0.2$ \\
\hline
\end{tabular}


Table S5. Transverse relaxation time $\left(\mathrm{T}_{2}\right)$ and peak amplitude of non-degraded and degraded corn stover anatomical fractions.

\begin{tabular}{lccccc}
\hline & $\begin{array}{c}\text { Anatomical } \\
\text { fractions }\end{array}$ & Pool $_{\mathrm{b}}(\mathrm{ms})$ & $\mathrm{A}_{\mathrm{b}}(\%)$ & Pool $_{\mathrm{f}}(\mathrm{ms})$ & $\mathrm{A}_{\mathrm{f}}(\%)$ \\
non-degraded & Cob & $0.66 \pm 0.005$ & $1.81 \pm 0.05$ & $200 \pm 10$ & $24.4 \pm 0.4$ \\
& Leaf & $1.42 \pm 0.009$ & $15.20 \pm 0.6$ & $74 \pm 9$ & $25.6 \pm 0.2$ \\
& Stalk & $170 \pm 30$ & $11.60 \pm 0.5$ & & \\
degraded & Cob & $150 \pm 20$ & $17 \pm 0.3$ & & \\
& Leaf & $0.80 \pm 0.007$ & $6.90 \pm 0.2$ & $50 \pm 10$ & $22.3 \pm 0.3$ \\
& Stalk & $150 \pm 30$ & $8.40 \pm 0.3$ & & \\
\hline
\end{tabular}


Table S6. Physicochemical property of the non-degraded and biologically degraded corn stover at different anatomical fractions ${ }^{1}$.

\begin{tabular}{llll}
\hline $\begin{array}{l}\text { Stover fraction and } \\
\text { degradation level }\end{array}$ & $\begin{array}{l}\text { Specific surface } \\
\text { area }\left(\mathrm{m}^{2} / \mathrm{g}\right)\end{array}$ & $\begin{array}{l}\text { Average pore } \\
\text { diameter }(\mathrm{A})\end{array}$ & $\begin{array}{l}\text { Pore volume*10 } \\
\left(\mathrm{cm}^{3} / \mathrm{g}\right)\end{array}$ \\
Cob, non-degraded & 0.53 & 180 & 1.60 \\
Cob, degraded & 0.49 & 330 & 1.50 \\
Leaf, non-degraded & 0.52 & 155 & 1.50 \\
Leaf, degraded & 1.20 & 185 & 4.75 \\
Stalk, non-degraded & 1.06 & 165 & 3.75 \\
Stalk, degraded & 1.04 & 190 & 4.00 \\
\hline
\end{tabular}


Table S7. Compositional analysis of corn stover anatomical fractions given as a percentage of the total mass.

\begin{tabular}{cccccccc}
\hline Samples & Glucan & Xylan & Arabinan & Acetate & $\begin{array}{c}\text { Acid- } \\
\text { insoluble } \\
\text { lignin }\end{array}$ & $\begin{array}{c}\text { Acid- } \\
\text { soluble } \\
\text { lignin }\end{array}$ & $\begin{array}{c}\text { Water } \\
\text { extractives }\end{array}$ \\
\hline Non-degraded, cob & 32.34 & 30.34 & 3.48 & 4.24 & 15.33 & 2.04 & 2.40 \\
Non-degraded, leaf & 34.90 & 18.10 & 4.41 & 1.03 & 11.94 & 1.39 & 0.99 \\
Non-degraded, stalk & 37.52 & 18.36 & 2.66 & 1.84 & 17.28 & 1.38 & 2.88 \\
Degraded, cob & 31.33 & 17.04 & 0.53 & 0.85 & 15.71 & 1.22 & 8.78 \\
Degraded, leaf & 31.64 & 10.77 & 0.50 & 0.48 & 18.23 & 1.12 & 9.72 \\
Degraded, stalk & 32.91 & 11.94 & 0.40 & 0.89 & 21.11 & 1.05 & 10.94 \\
\hline
\end{tabular}




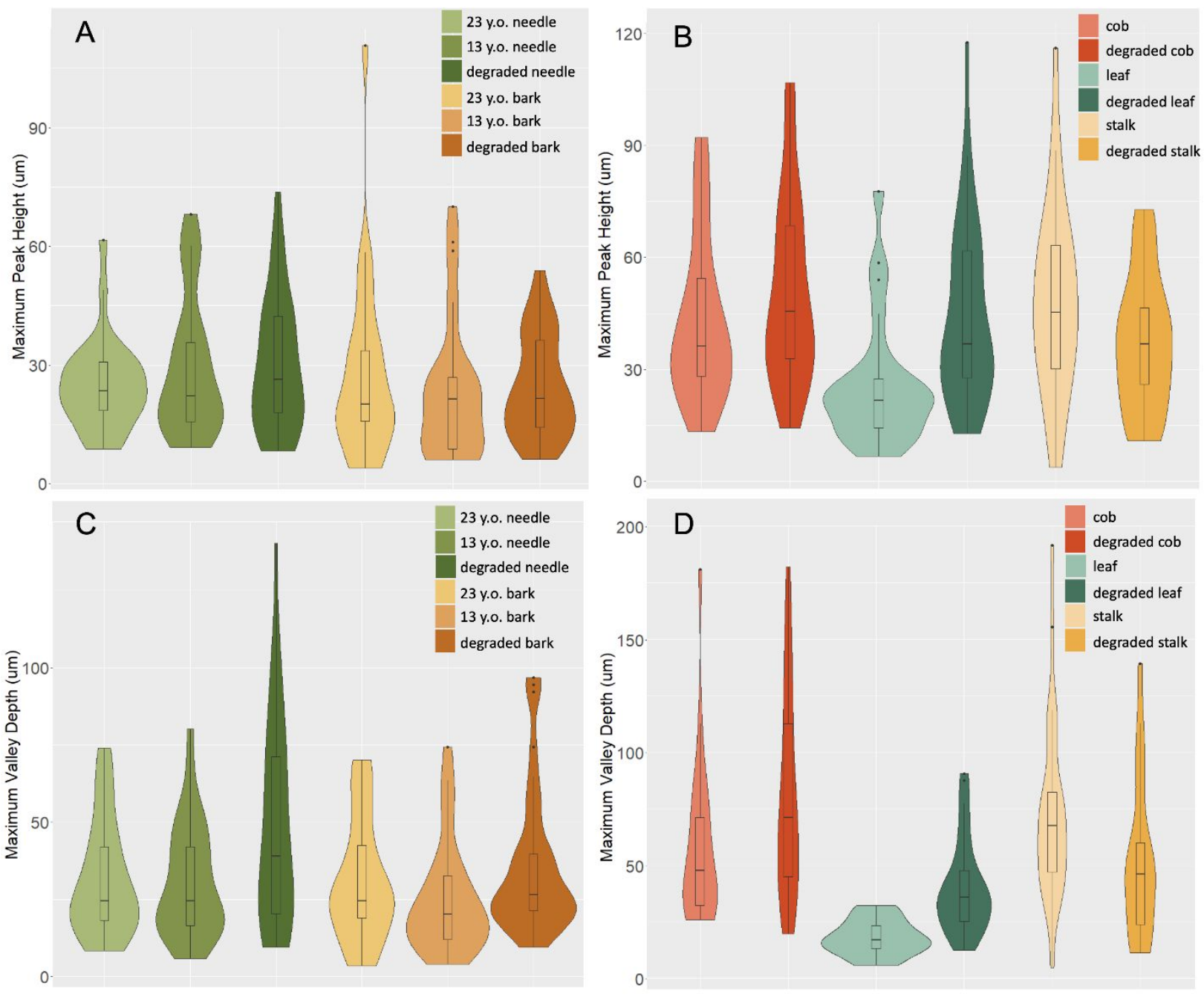

Figure S1. Distribution of maximum peak height (Sp) calculations of corn stover (A) and pine residue (B) material and maximum valley depth (Sv) of corn stover (C) and pine residue (D) extracted from 3D stereometric reconstructions. Violin plots of Sp and Sv value distributions and overlaid boxplots indicate that degradation increases surface heterogeneity, as well as surface roughness. 


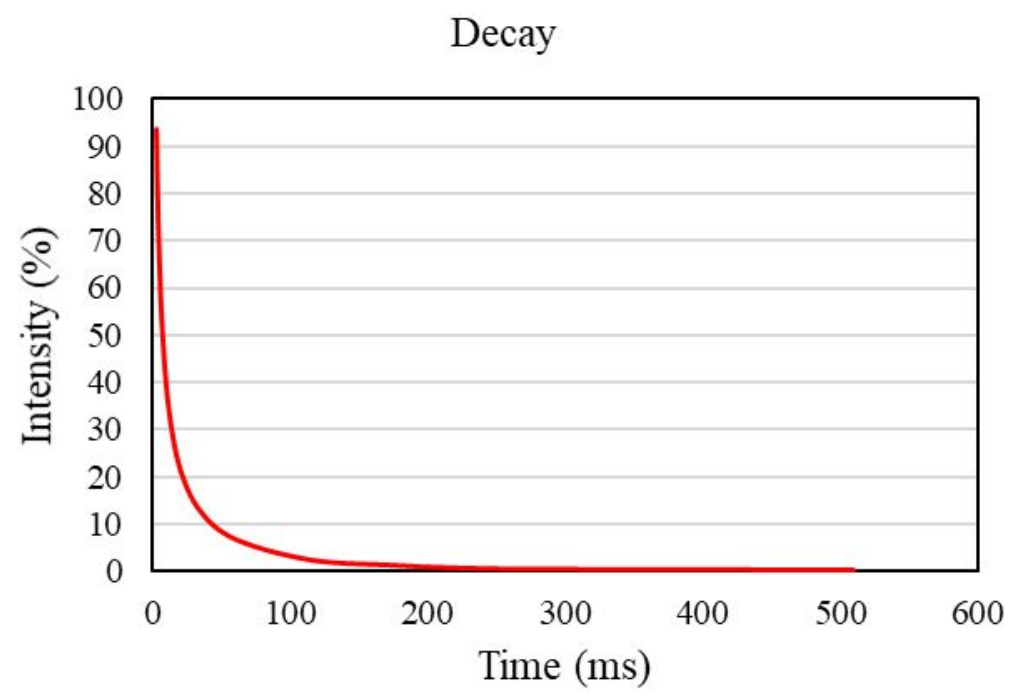

Figure S2. Raw, non-Laplace transformed decay of 23-year-old needle fractions.

1. Bose, E., Leal, J. H., Hoover, A. N., Zeng, Y., Li, C., Ray, A. E., ... \& Donohoe, B. S., Impacts of Biological Heating and Degradation during Bale Storage on the Surface Properties of Corn Stover. Acs Sustain Chem Eng August 13, 2020, 8(37), 13973-13983. 